\title{
Allocating Bandwidth Using Fair Random Indication with Discarding Aggressiveness Yardstick (FRIDAY)
}

\author{
Shushan Wen, Hairong Sun, Lemin Li \\ National Key Lab of Optical Fiber Transmission and Communication Networks, University of \\ Electronic Science and Technology of China. Email: wenss@uestc.edu.cn \\ System Engineering Technology Center, Motorola.Email:ahs013@email.mot.com \\ National Key Lab of Optical Fiber Transmission and Communication Networks, University of \\ Electronic Science and Technology of China. Email: lml@uestc.edu.cn
}

\begin{abstract}
In order to improve the fairness of bandwidth allocation in the Internet, we present an active queue management scheme called Fair Random Indication with Discarding Aggressiveness Yardstick (FRIDAY), and study its performance via combining it with certain scheduling algorithms, e.g., FCFS (First-Come-First-Served) and FQ (Fair Queueing). By calculating the average arriving rate of each flow or a set of merged flows based on EWMA (Exponentially Weighted Moving Average), and discarding packets from the most aggressive flow or flow set randomly according to a dropping probability, FRIDAY is able to (1) provide better fairness than LQD (Longest Queue Drop), RED (Random Early Detection), and TD (Tail Drop), (2) maintain a shorter average waiting time in the queue than LQD and TD, and (3) control the time limit for a bursty traffic to send data continuously without packet dropping. The performances of FRIDAY are validated by $n s$ simulator.
\end{abstract}

\section{INTRODUCTION}

In the Internet, bandwidth allocation algorithm must be examined together with the end-to-end congestion control mechanisms (e.g., TCP) and the buffer management schemes implemented in the routers or switches, in order to get a complete picture on its performance impact to the whole system. If we construct a two-dimensional plane with the $X$-axis representing the dropping location in the queue (i.e., from where a packet is going to be dropped) and $Y$-axis representing the dropping time (i.e., at when a packet is determined to be dropped) (as shown in Figure 1), any buffer management algorithm would be mapped into a certain area of this plane. For the sake of the wide use of packet discarding as the method to manage the buffers in today's Internet, the axes in Figure 1 are given the name as dropping time and dropping location. Nevertheless, we do not exclude the cases of marking a packet instead of dropping it in tomorrow's network.

From the viewpoint of dropping time, a buffer management algorithm is called "full-discard" if the buffer discards a packet when it is full upon a new arrival. Otherwise, the algorithm dropping packets before the buffer overflow occurs is called "early-discard". From the viewpoint of dropping location, an algorithm can be

This work is supported by National Natural Science Foundation of China (No. 69882003)

The original version of this chapter was revised: The copyright line was incorrect. This has been corrected. The Erratum to this chapter is available at DOI: 10.1007/978-0-387-35620-4_43 
categorized into front drop, tail drop, or random drop according to the location from which a packet is discarded - the front, the tail, or a random place of the queue.

The simplest buffer management algorithm is Tail Drop (TD). The conventional TD, i.e. Tail Drop on Full, is mapped into rectangular area $G K L H$ in Figure 1. It was found that TD allows a single flow or a few flows to monopolize queue space (i.e., the "lock-out" phenomenon) and maintains a status of almost full queue for long periods of time [1]. Random Drop on Full and Front Drop on Full [2], which are mapped into rectangular

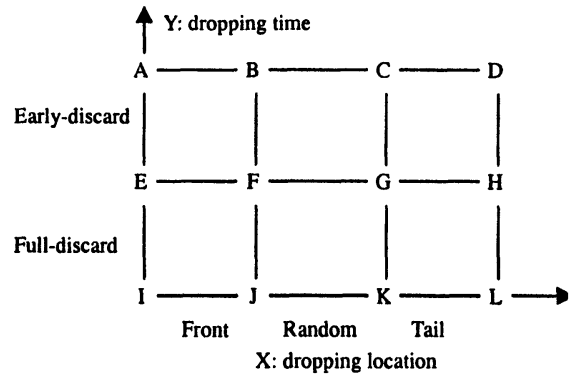

Figure 1. The plane of buffer management categories areas $F J K G$ and $E I J F$, respectively, are improvements to the conventional TD and able to address the "lock-out" problem. But neither solves the full-queue problem [1]. All the algorithms in rectangular area EILH cannot provide desirable features like shorter queue and waiting time. Another category of buffer management schemes is called Active Queue Management (AQM), which is mapped into area $A E H D$ in Figure 1. Random Early Detection (RED) [3] is the most widely studied $\mathrm{AQM}$. By discarding packets according to a calculated dropping probability before the queue is full, RED provides several nice features such as maintaining a shorter average queue length and avoiding the global synchronization to some extent. The original RED in [3] is mapped into rectangular area $C G H D$, but RED itself can be mapped into $A E F B$ and $B F G C$ as well. In [4], RED with TD was recommended due to its simplicity and straightforwardness if the average queue length is short.

RED, however, is not able to provide satisfactory fairness, especially when the RTTs (Round-Trip-Times) of the TCP connections differ significantly. The sending rate of a TCP connection is inversely proportional to its RTT approximately, supposing all the connections encounter the same dropping probability $[5,6]$. Therefore, the TCP connection with a longer RTT will get a lower bandwidth from a RED router.

In order to improve the fairness, Longest Queue Drop (LQD) was presented in [7]. The spirit of LQD is to drop the front packet from the queue that occupies the largest portion of the buffer space. However, since the burstiness of the traffic in the Internet, the flow occupying the most buffer space is not necessarily the one with the highest sending rate. Therefore, LQD has a bias against the traffic that is bursty but has a low average sending rate. Moreover, LQD is still a "full-discard" scheme and shares the disadvantages with all the other schemes in area EILH, e.g., full-queue problem and longer end-to-end delay.

In order to furthur improve the fairness, at the same time to trim the end-to-end delay, we present a new AQM scheme called Fair Random Indication with Discarding Aggressiveness Yardstick (FRIDAY). FRIDAY integrates the benefits of flow isolation (like LQD) and early discard (like RED). It is mapped into the area $A E H D$ in Figure 1. 
In order to analyze the performance of FRIDAY, we study the goodput, the fairness and the average waiting time in the queue by combining it with scheduling algorithms like First-Come-First-Served (FCFS) and Fair Queueing (FQ). The performances of FRIDAY are compared with the other buffer management schemes, including TD, LQD, and RED.

In Section 2, we discuss the principles of FRIDAY algorithm. In Section 3, we explain how to regulate the time limit to detect the most aggressive flow by setting the weights in Exponentially Weighted Moving Average (EWMA). The results of our simulations based on $n s$ and its revised version with FRIDAY algorithm are given in Section 4. The simplification of implemention is discussed in Section 5. Finally, conclusions are drawn in Section 6. Further possible extensions are also discussed in Section 6.

\section{THE PRINCIPLES OF FRIDAY}

\subsection{Fuction Modules}

FRIDAY is a scheme that supports shared buffer but permits per-flow queueing. It calculates the arriving rate for each flow and discards the packets from the most aggressive flow randomly when the congestion occurs or will occur. The function modules of FRIDAY are shown in Figure 2. Conceptually, FRIDAY consists of two function modules called Arriving-Rate Counter and Dropping Judge. These two modules are responsible to control the allocation of buffer space and give a congestion indication to a certain flow by dropping an appropriate packet.

As a buffer management scheme, the performance of FRIDAY will be affected by the algorithm implemented in the scheduler. Since FRIDAY and scheduling algorithm are independent of each other conceptually, the scheduler is not included in FRIDAY. When a new packet arrives, the classifier, which is independent of FRIDAY as well, checks the

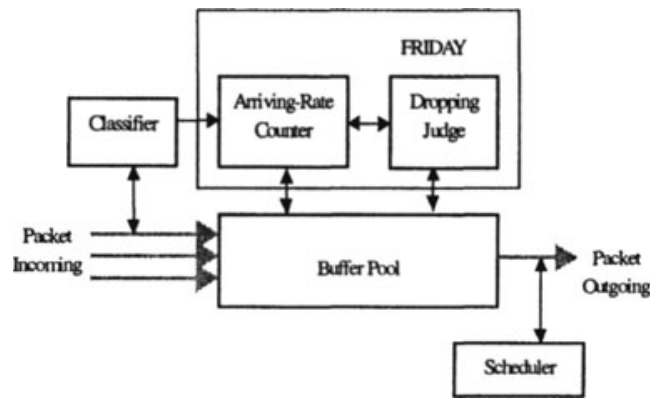

Figure 2. Function modules of FRIDAY related fields in the header of the arriving packet, e.g., the source address and the destination address, and classifies it into a flow that is represented by an ID in the router. As a matter of fact, the buffer management scheme and the scheduling algorithm that deal with a flow can be used to deal with a flow set as well. When the number of active connections expands, or the capability of the router is too low to meet the demand of per-flow operation, merging the flows that share certain attributions into a flow set is a wise choice. In this case, all the flows in the set are represented as a whole by an ID in the router.

After it is classified, the arriving packet will enter the logical queue related to the flow or flow set. Then, the Arriving-Rate Counter in FRIDAY will calculate the 
arriving rate of the flow. If necessary, the Dropping Judge in FRIDAY will discard a packet from the most aggressive flow.

\subsection{Principles and Explanations}

The principles of FRIDAY are listed as follows:

(1) If necessary, FRIDAY will discard a packet in the buffer pool from the most aggressive flow, i.e., the flow with the highest arriving rate. If the most aggressive flow does not have a packet in the buffer pool - although this is not an ordinary case - FRIDAY will discard a packet in the buffer pool from the flow with the 2nd highest arriving rate, and so on. This function is implemented in the Dropping Judge module based on the information provided by the Arriving-Rate Counter.

(2) FRIDAY refreshes the arriving rate of each flow after every time interval $\tau$, and the flows are ordered according to their arriving rate, from the highest to the lowest. This is implemented in the Arriving-Rate Counter.

(3) Enqueue the arriving packet at first. Then, FRIDAY calculates a dropping probability dependent on the status of buffer occupancy, and resorts to Principle (1). This is controlled by the Dropping Judge module.

Now we come up with some explanations on the above principles.

Principle (1) only determines in which queue packet will be dropped (i.e., the queue of the most aggressive flow), and does not define the dropping location (front, tail, or random) in the queue of the most aggressive flow. That means FRIDAY can employ Tail Drop, Front Drop, or Random Drop as you like. In our simulations, we use Front Drop for the same reason as in LQD, i.e., decreasing the response delay of TCP connections as short as possible.

Take note of the fact that both FRIDAY and FQ are per-flow mechanisms, while FCFS is not. Therefore, when we use FRIDAY combined with FQ (FQFRIDAY), we are in a dilemma about making a choice between a shared queue for FCFS and multiple queues for per-flow queueing. A special data structure, i.e., a linked list or a group of linked lists can be constructed as a virtual queue or virtual queues in the router to handle it. Each link element is a pointer to the location in which a packet is stored in the buffer, i.e., the content of the link element is an address, not a packet.

Under the constraint of Principle (2), FRIDAY calculates the arriving rate for each flow based on EWMA. It can improve fairness, especially correct the bias against the flows with lower average sending rate but higher burstiness. This idea is used in $[8,9]$ with a variable of EWMA weight depending on the inter-arrival time of packets from a certain flow. In $[8,9]$ the rate record of a flow is updated upon every arrival, but the calculation implemented in FRIDAY is much simpler due to the fixed EWMA weight value and the operation that the rate needs only updating at time $n \tau$.

For flow $i$, let $R_{i}(n)$ represent the observed arriving rate in $n$th interval $\tau$, and let $M_{i}(n)$ represent the calculated average arriving rate at the end of $n$th interval $\tau$, i.e., time $n \tau$. Using the method of EWMA, we have:

$$
M_{i}(n)=(1-w) M_{i}(n-1)+w R_{i}(n) .
$$


In order to increase the calculation speed and to implement the calculation by hardware, the EWMA weight, $w(0<w<1)$, can be assigned with a value as $2^{-k}(k$ is a positive integral number). Then the multiplicative operations are replaced by shifts.

In order calculate the arriving rate for each flow, we define a variable $B_{i}$ for flow $i$, which has an initial value 0 . When the classifier categorizes the new-arriving packet into flow $i$, the size of this packet (i.e., number of bytes) will be added to $B_{i}$. When FRIDAY discards a packet from flow $i$, the packet size will be deducted from $B_{i}$, because this packet will not occupy the downstream bandwidth. After time interval $\tau$, the value of $B_{i}$ equals the total length of packets from flow $i$ that have occupied or will occupy the downstream bandwidth. Let $B_{i}(n)$ represent the value of $B_{i}$ at time $n \tau$. Then we have

$$
M_{i}(n)=(1-w) M_{i}(n-1)+w B_{i}(n) / \tau .
$$

After calculating the average arriving rate $M_{i}$ for flow $i$ each time interval ${ }^{1}$, the Arriving-Rate Counter in FRIDAY will reset $B_{i}$ to 0 , and continue its next calculation. If flow $i$ has stopped sending data, the value of $M_{i}$ will decrease gradually. Once it is smaller than a pre-defined threshold, FRIDAY will regard flow $i$ as silent and release the variables reserved for it (i.e., $B_{i}$ and $M_{i}$ ). In our simulations, a threshold of 40 bytes $/ \tau$ is chosen, which equals only one ACK packet during interval $\tau$. And we set $\tau=2 d$, where $d$ denotes the propagation delay of the downstream link (its approximate value can be obtained by the command "ping" when the link is idle and the network configurations are initialized by the administrator). Thus, all the values of RTTs of the connections through the link are larger than $\tau$.

It is worthy of note that FRIDAY pays more attention to the aggressive flows, and the threshold used to release the variables $B_{i}$ and $M_{i}$ described above could be any value smaller than the arriving rate of most flows.

In Principle (3), FRIDAY is similar to RED and discards packets randomly before the buffer is full. But FRIDAY is different from RED in two aspects: (1) Random dropping in RED is imposed on all the flows while FRIDAY only discards the packets from the most aggressive flow. (2). EWMA in RED is used to calculate the average queue length avg, which determines the dropping probability, while in FRIDAY, EWMA is used to calculate the average arriving rate $M_{i}$, and the dropping probability is determined by the current status of buffer occupancy (i.e., the total length of all the queues in the buffer pool at the present time, rather than the average status of buffer occupancy). In this paper, we do not smooth the queue length in FRIDAY to absorb the low-rate but bursty traffic, since this kind of traffic is absorbed by the buffer when the arriving rate is smoothed. One way to set the dropping probability $p$ in FRIDAY is shown as follows:

$$
p=\left\{\begin{array}{ccc}
0 & , \quad L \leq L_{\min } \\
\frac{L-L_{\min }}{L_{\max }-L_{\min }} \cdot p_{\mathrm{th}}, & L_{\min }<L \leq L_{\max }, \\
1, & L>L_{\max }
\end{array}\right.
$$

\footnotetext{
${ }^{1}$ Strictly, because of the method used to calculate $B_{i}$, the value of $M_{i}$ equals the average downstream bandwidth allocated to flow $i$.
} 
where $L, L_{\min }, L_{\max }, p_{\mathrm{th}}$ are similar to qlen (not $a v g$ ), $\min _{\mathrm{th}}, \max _{\mathrm{th}}, \max _{\mathrm{p}}$ in RED. However, it should be clarified that Equation (3) is not the only method to set the value of $p$. Any other expressions of $p$ being able to reflect the severity of the network congestion are also candidates.

We ran the simulations for FRIDAY with and without EWMA queue length separately, and got the results of goodput, link utilization, fairness and average waiting time in the queue (not listed in this paper). It was found that the performance difference of the two FRIDAYs are insignificant.

When the number of TCP connections is large, we adopt the fairness coefficient

$$
F=\frac{\left(\sum_{i=1}^{N} G_{i}\right)^{2}}{N \sum_{i=1}^{N} G_{i}{ }^{2}}
$$

to qualify the fairness, where $N$ denotes the number of active flows during the observation period, and $G_{i}(i=1,2, \ldots, N)$ represents the goodput of flow $i$.

\section{TIME TO DETECT THE MOST AGGRESSIVE FLOW}

FRIDAY is able to control the time to detect the most aggressive flow by setting the value of EWMA weight $w$ in equation (2). In order to give an analytical explanation, let us observe flow $i$ at first. Suppose the observation begins at time 0 and the average arriving rate of flow $i$ is $R_{i}$, i.e., $M_{i}(0)=R_{i}$. After time 0 , flow $i$ increases its rate to reach $R_{i}^{\prime}\left(R_{i}^{\prime}>R_{i}\right)$. Then, the refreshed average arriving rate of flow $i$ at time $\tau$ is

$$
M_{i}(1)=(1-w) R_{i}+w R_{i}^{\prime} .
$$

When it is refreshed at time $2 \tau$,

$$
M_{i}(2)=(1-w) M_{i}(1)+w R_{i}^{\prime}=(1-w)^{2} R_{i}+w(1-w) R_{i}^{\prime}+w R_{i}^{\prime} .
$$

Generally, at time $n \tau(n=1,2, \ldots)$, the average arriving rate is

$$
M_{i}(n)=R_{i}(1-w)^{n}+R_{i}^{\prime} w \sum_{k=0}^{n-1}(1-w)^{k}=R_{i}(1-w)^{n}+R_{i}^{\prime}\left[1-(1-w)^{n}\right] .
$$

Let $R_{i}^{\prime}=R_{i}+\Delta R_{i}$, we have

$$
M_{i}(n)=R_{i}+\Delta R_{i}-\Delta R_{i}(1-w)^{n} .
$$

Supposing flow $j$, with the arriving rate of $R_{j}\left(R_{i}<R_{j}<R_{i}^{\prime}\right)$, was the most aggressive flow at time 0 and maintains its arriving rate, i.e.,

$$
M_{j}(n)=M_{j}(n-1)=\ldots=M_{j}(0)=R_{j} .
$$

Denote the time needed for flow $i$ to catch up with flow $j$ in the sense of average arriving rate as $n \tau$. Then $n$ should satisfy

$$
R_{i}+\Delta R_{i}-\Delta R_{i}(1-w)^{n} \geq R_{j}
$$




$$
n \geq \log _{1-w} \frac{R_{i}+\Delta R_{i}-R_{j}}{\Delta R_{i}} .
$$

Equation (11) gives the time needed for a less aggressive flow to catch up with and then replace the original most aggressive flow in the sense of average arriving rate. That is also the time limit for a bursty traffic to send data continuously without packet dropping. Especially, if $R_{i}=0, \Delta R_{i}=R_{i}^{\prime}$, Equation (10) can be rewritten as

$$
n \geq \log _{1-w}\left(1-\frac{R_{j}}{R_{i}^{\prime}}\right) \text {. }
$$

Since $0<w<1$, after $w$ has been determined, the smaller $R_{j}$ $/ R_{i}^{\prime}$ is, the smaller $n$ is. From another point of view, after $R_{j}$ $/ R^{\prime}{ }_{i}$ has been determined, the larger $w$ is, the smaller $n$ is. Smaller value of $n$ means that it needs shorter time for flow $i$ to take the place of flow $j$ and become the most aggressive flow. It infers that FRIDAY is quicker to detect the new aggressive flow if it is assigned with a larger $w$. The relationship among $n, R_{j} / R_{i}^{\prime}$

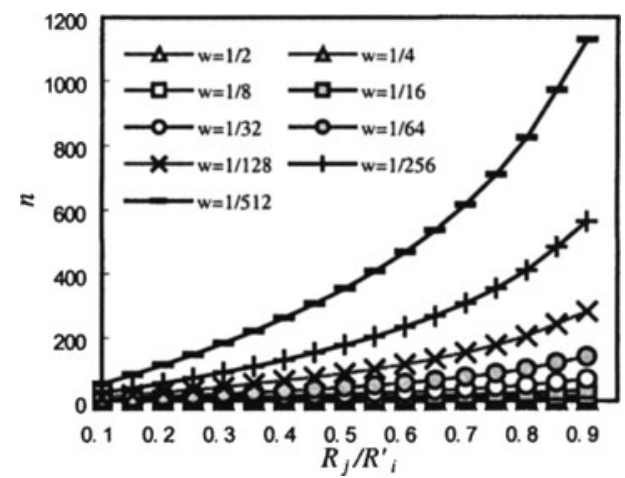

Figure 3. The relationship among $n, R_{j} / R_{i}^{\prime}$ and $w$ and $w$ is shown in Figure 3.

\section{SIMULATIONS}

In order to compare the combinations of various schedulers (FCFS and FQ) and buffer management schemes (TD, LQD, RED and FRIDAY), we add the source code of FRIDAY and LQD into $n s$ simulator version 2.1b6 [10].

In this paper, $L_{\min }$ of FRIDAY and $\min _{\mathrm{th}}$ of RED are set as $1 / 3$ of the buffer size, $L_{\max }$ and $\max _{\text {th }}$ are set as $2 / 3$ of the buffer size, and $p_{\text {th }}$ and $\max _{\mathrm{p}}$ are 0.002 . RED has the EWMA weight as 0.002 to calculate $a v g$, and runs in byte mode with the average packet size of 1000 bytes. The simulation network is illustrated in Figure 4, with the EWMA weight of FRIDAY being set as $w=2^{-8}=1 / 256$.

\subsection{Two TCP Flows}

In this experiment two TCP flows are used to transmit FTP traffic, whose sources are $S_{1}$ and $S_{2}$, and destinations are $D_{1}$ and $D_{2}$ respectively. The link between $R_{1}$ and $R_{2}$, with the bandwidth of 10 $\mathrm{Mb} / \mathrm{s}$ and the propagation delay of 1 millisecond, is the bottleneck link.

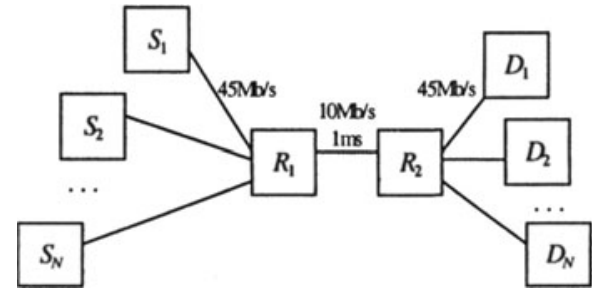

Figure 4. The simulation network 
The end-to-end propagation delay from $S_{1}$ to $D_{1}$ (i.e., the sum of all the propagation delay of the links on the path from the source node $S_{1}$ to the destination node $D_{1}$ ) is $10 \mathrm{~ms}$. The end-to-end propagation delay from $S_{2}$ to $D_{2}$ is $50 \mathrm{~ms}$. The size of the buffer pool is 120 packets.

Different versions of TCP (Tahoe, Reno, Newreno, and SACK) are used to repeat the simulation, and every simulation runs for 100 seconds. The results of goodput, link utilization (ratio of the aggregate throughput to the bandwidth of the bottleneck link) and the average waiting time for both flows are listed in Table 1, 2 and 3 respectively (T, R, N and S denotes Tahoe, Reno, Newreno and SACK respectively). The average waiting time is expressed in terms of the number of average-sized (1000-bytes) packets that would be forwarded during the time period.

From Table 1, one can find that FRIDAY provides better fairness than LQD, TD and RED. Generally FQ scheduler provides more fair resource sharing than FCFS except in some cases, e.g., FQ-RED is not better than FCFS-RED. The exceptional phenomenon is attributed to the fact that RED is not able to provide isolation between flows. Table 2 demonstrates that the link utilization of FRIDAY is not worse than any other algorithms. In Table 3 , for a certain buffer management scheme, FCFS scheduling algorithm provides very similar average waiting time to different flows, since the packets from different flows share a single logical queue. In contrast to that, $\mathrm{FQ}$ scheduler will lower the average waiting time of the flow with the larger RTT because of its lower sending rate. From Table 3, it is also observed that the average waiting time of TD and LQD is much longer than that of RED and FRIDAY:

Table 1. Goodput of two TCP flows (Mb/s)

\begin{tabular}{|c|c|c|c|c|c|c|c|c|}
\hline & \multicolumn{2}{|c|}{ TD } & \multicolumn{2}{c|}{ RED } & \multicolumn{2}{c|}{ LQD } & \multicolumn{2}{c|}{ FRIDAY } \\
\cline { 2 - 9 } & FCFS & FQ & FCFS & FQ & FCFS & FQ & FCFS & FQ \\
\hline T & $8.96 / 0.71$ & $7.75 / 2.02$ & $7.05 / 2.66$ & $7.16 / 2.15$ & $6.31 / 3.39$ & $5.69 / 4.10$ & $5.94 / 3.78$ & $5.65 / 4.19$ \\
\hline R & $9.15 / 0.38$ & $8.07 / 1.62$ & $9.69 / 0.15$ & $9.69 / 0.18$ & $7.99 / 1.46$ & $6.17 / 3.52$ & $7.82 / 1.75$ & $6.13 / 3.56$ \\
\hline $\mathrm{N}$ & $9.15 / 0.74$ & $7.98 / 1.97$ & $7.10 / 2.86$ & $7.44 / 2.42$ & $6.43 / 3.29$ & $5.73 / 4.06$ & $6.46 / 3.50$ & $5.69 / 4.30$ \\
\hline $\mathrm{S}$ & $9.17 / 0.79$ & $7.28 / 2.68$ & $7.32 / 2.67$ & $6.67 / 3.23$ & $6.42 / 3.54$ & $5.92 / 4.06$ & $5.76 / 4.23$ & $5.15 / 4.83$ \\
\hline
\end{tabular}

Table 2. Link Utilization (\%)

\begin{tabular}{|c|c|c|c|c|c|c|c|c|}
\hline \multirow{2}{*}{} & \multicolumn{3}{|c|}{ TD } & \multicolumn{2}{c|}{ RED } & \multicolumn{2}{c|}{ LQD } & \multicolumn{2}{c|}{ FRIDAY } \\
\cline { 2 - 9 } & FCFS & FQ & FCFS & FQ & FCFS & FQ & FCFS & FQ \\
\hline T & 96.8 & 97.9 & 97.2 & 93.2 & 97.1 & 98.0 & 97.4 & 98.5 \\
\hline R & 95.5 & 97.0 & 98.5 & 98.7 & 94.7 & 97.0 & 95.9 & 97.1 \\
\hline N & 99.0 & 99.6 & 99.6 & 98.6 & 97.3 & 98.1 & 99.7 & 99.9 \\
\hline S & 99.6 & 99.7 & 99.9 & 99.0 & 99.6 & 99.8 & 99.9 & 99.8 \\
\hline
\end{tabular}

Table 3. Average waiting time in the queue (packets)

\begin{tabular}{|c|c|c|c|c|c|c|c|c|}
\hline \multirow{2}{*}{} & \multicolumn{2}{|c|}{ TD } & \multicolumn{2}{c|}{ RED } & \multicolumn{2}{c|}{ LQD } & \multicolumn{2}{c|}{ FRIDAY } \\
\cline { 2 - 9 } & FCFS & FQ & FCFS & FQ & FCFS & FQ & FCFS & FQ \\
\hline T & $40.5 / 41.2$ & $69.3 / 2.7$ & $31.0 / 31.6$ & $46.3 / 2.9$ & $40.2 / 39.0$ & $66.1 / 14.9$ & $26.8 / 27.7$ & $38.0 / 7.9$ \\
\hline $\mathrm{R}$ & $35.6 / 39.2$ & $44.7 / 2.2$ & $24.4 / 24.1$ & $34.0 / 2.0$ & $35.0 / 35.1$ & $50.7 / 24.7$ & $21.1 / 22.1$ & $36.6 / 22.9$ \\
\hline $\mathrm{N}$ & $42.2 / 42.0$ & $38.3 / 5.1$ & $27.1 / 27.3$ & $28.9 / 8.8$ & $40.7 / 40.1$ & $32.5 / 10.7$ & $21.0 / 19.1$ & $36.9 / 9.2$ \\
\hline $\mathrm{S}$ & $40.9 / 41.4$ & $59.5 / 9.5$ & $32.5 / 33.0$ & $45.4 / 2.4$ & $39.2 / 37.8$ & $55.5 / 34.4$ & $30.3 / 30.4$ & $43.8 / 24.7$ \\
\hline
\end{tabular}

Considering the fact that the traffic transported by TCP mainly belongs to nondelay-sensitive traffic rather than real time traffic, we regard the fairness and goodput as the principal criterion, and the average waiting time in the queue as the 
secondary one. Therefore, in the case of 2 TCP flows, FRIDAY has the best performance among the four buffer management schemes.

\subsection{UDP and TCP Competing for Bandwidth}

In this subsection, we study the performance of FRIDAY in the case of UDP competing bandwidth with TCP. One couple of nodes, $S_{i}$ and $D_{i}(i=1,2, \ldots, 11)$, generates one flow. In these 11 flows, 10 flows $(i \leq 10)$ are TCP and one flow is UDP $(i=11)$, and their end-to-end propagation delays are in the range of 5 to $55 \mathrm{~ms}$. The 10 TCP flows consist of 2 Tahoe, 2 Reno, 3 Newreno and 3 SACK. TCP is

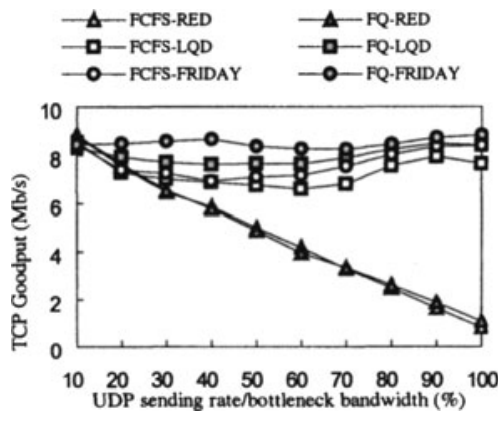

Figure 5. TCP goodput influenced by

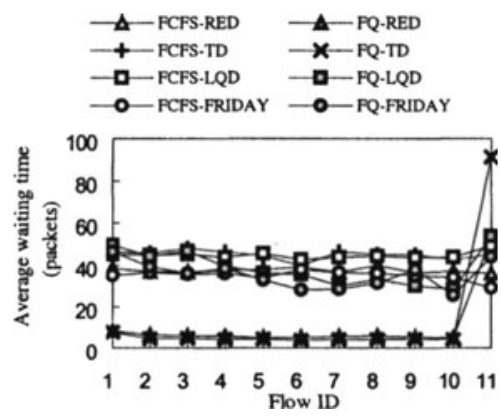

Figure 6. Average waiting time of each

driven by FTP, and UDP is driven by CBR (Constant Bit Rate). The buffer size equals 120 packets. Each simulation result is represented by one point in Figure 5.

One of the observations can be made from Figure 5 is that there is an approximately linear relationship between the aggregate TCP goodput and the UDP sending rate in FCFS-RED and FQ-RED. As a typical unresponsive flow, UDP does not reduce its sending rate even though its packets are dropped because of network congestion. Therefore, when the TCP flow cuts its rate, the saved bandwidth will be occupied by the UDP flow soon. In FRIDAY and LQD, however, this problem does not occur because of the flow isolation function supported by both schemes. Moreover, with the same scheduler, TCP goodput in FRIDAY is better than that in LQD, because the bursty traffic can be absorbed by the buffer of FRIDAY that use EWMA to smooth the arriving rate, while in LQD, the bursty traffic might cause itself to become the flow that has the longest queue in the buffer and hence experiences packet dropping.

We further study the average waiting time in the queue for each flow with the UDP flow rate of $6 \mathrm{Mb} / \mathrm{s}$ (i.e., $60 \%$ of the bottleneck bandwidth). The simulation results are shown in Figure 6, where the TCP flows are represented by the flow IDs from 1 to 10 and the UDP flow is represented by ID 11. The TCP flows obtain shortest average waiting time in FQ-TD and FQ-RED, but the performance is still not satisfactory because of the low goodput (Please refer to Figure 5 at the same time.).

Due to the nature of randomness in the network, when the number of flows is large, we cannot guarantee that all the flows experience shorter average waiting time in one scheme than that in the other schemes. The scheme being capable of 
providing most of the flows with shorter average waiting time is better. Consequently, FRIDAY is also better than LQD when same scheduler is employed.

In Figure 6, one can also find that the average waiting time of the flow with ID 11 (UDP) is much longer than that of the other flows in FQ-RED and FQ-TD. The cause to this phenomenon is that the high-rate UDP flow occupies a much larger space in the buffer pool than any TCP flow does. When FQ scheduler is used, the UDP packets in the buffer form a logical queue much longer than any logical queue relative to a TCP flow.

\subsection{ON-OFF Pareto Sources Mixed with FTP}

A great number of researches manifested that the traffic in the Ethernet and the Wide Area Networks (WANs) can be characterized by self-similarity [11 13]. The packets of the TELNET traffic generated by a keyboard follows Pareto distribution
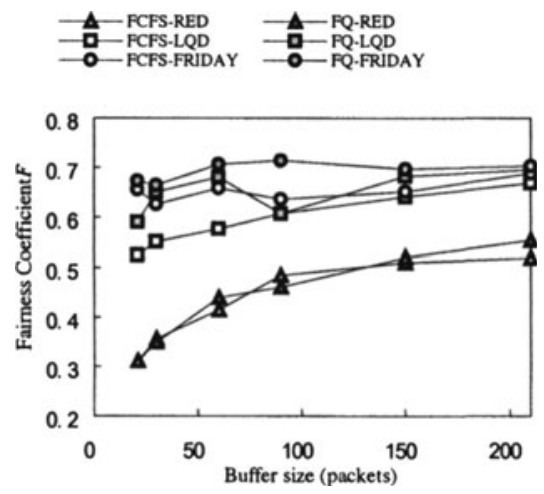

Figure 7. Relationship between $F$ and buffer size in the circumstance of ON-OFF Pareto sources mixed with FTP (The number of TCP flows $=44$ )

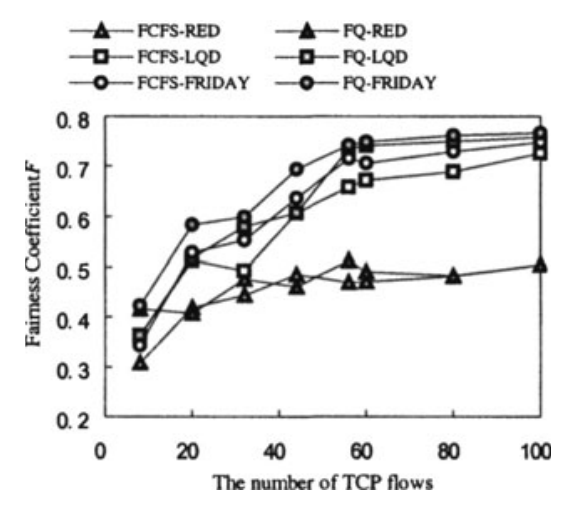

Figure 8. Relationship between $F$ and the number of TCP flows in the circumstance of ON-OFF Pareto sources mixed with FTP (Buffer size $=90$ packets)

[11], and WWW traffic complies with Pareto distribution with 1.16<o<1.5 [12].

We assume the TELNET applications have the parameters as follows: $\alpha=1.2$, fifteen 80-byte packets on the average being sent out during an ON period, and the average duration of OFF period being 1 second. We name it as Pareto ON-OFF sources Group 1 in this paper. The parameters for the WWW applications are as follows: $\alpha=1.4$, one hundred 1000-byte packets on the average being sent out during an $\mathrm{ON}$ period, and the average duration of OFF period being 3 second. We named it as Pareto ON-OFF sources Group 2. The number of flows driven by FTP, Pareto ON-OFF sources Group 1, and Pareto ON-OFF sources Group 2 stays in the proportion of 1:1:2.

In the case of 44 flows, the results of the fairness coefficient $F$ affected by the buffer size are shown in Figure 7. $F$ versus the total number of flows is given in Figure 8. Both Figure 7 and Figure 8 validate that the fairness of FRIDAY is better than LQD and RED. Referring to the simulation results in subsection 4.1 and 4.2, it is easy to find that the ON-OFF Pareto sources deteriorate the fairness. 


\section{CONSIDERATION FOR IMPLEMENTATION}

FRIDAY requires per-flow queueing, which is a time-consuming action for the router. For implementation, the operation of FRIDAY can be simplified in two aspects as follows:

(1) The Internet could be divided into peripheral network and core network. In the peripheral network, the number of flows is not very large, hence FRIDAY for per-flow queueing is recommended. In the core network, since there are enormous flows through it, merging a group of flows into a flow set is a possible solution. In this case, FRIDAY provides the flow sets, rather than the individual flows, with fairness.

(2) In Principle (2) described in Subsection 2.2, in order to detect the most aggressive flow and drop its packet, the flows are ordered according to their arriving rate after every time interval $\tau$. One simplified version of FRIDAY is just to order a few top aggressive flows (say top $M$ ), not all the flows.

Let us examine one of the top $M$ flows. Denote $q$ as the probability of a flow that has no packet in the buffer. Suppose the TCP window size is $K(K>0)$ times the buffer size $B$, and let $D$ represent the average waiting time in the queue (in averagesized packets), $T$ represent the RTT, and $b$ represent the link bandwidth. The probability that the flow has packet in the buffer (i.e. $1-q)$ is

$$
1-q>\frac{1}{T} \cdot \frac{K B+D}{b} .
$$

Note that FRIDAY only drops packet when $L>L_{\min }$ as described in Equation (3). Therefore, when dropping is decided, $D>L_{\min }$. Then the probability of the exceptional case (i.e., there are no packets of the top $M$ flows in the buffer) is

$$
Q=q^{M}<\left(1-\frac{1}{T} \cdot \frac{K B+L_{\min }}{b}\right)^{M} .
$$

Let

$$
Q_{\max }=\left(1-\frac{1}{T} \cdot \frac{K B+L_{\min }}{b}\right)^{M},
$$

we have

$$
M=\log _{\left(1-\frac{1}{T} \frac{K B+L_{\min }}{b}\right.} Q_{\max } .
$$

Based on Equation (15), one can decide at least how many most aggressive flows need ordering.

\section{CONCLUSIONS AND FUTURE WORK}

As an Active Queue Management, FRIDAY can be employed by routers, switches or other packet-forwarding equipments. FRIDAY can provide a better fairness than TD, RED and LQD, at the same time guarantee satisfactory link utilization and maintain a shorter average waiting time in the queue than LQD. The performance was validated in various simulation circumstances. In addition to the case of pure TCP, it was also validated that FRIDAY can provide protection for TCP goodput when there are high-rate and unresponsive UDP flows in the network 
competing for bandwidth. We also found that FQ-RED displays worse fairness than FCFS-RED in some cases.

In this paper, FRIDAY calculates the EWMA arriving rate for different flows with the same weight $w$. Recalling the feature in Figure 3, i.e., the larger $w$ is, the quicker for FRIDAY to detect the new aggressive flow, a promising extension of FRIDAY is to introduce priority level and use different weight $w$ for the flows at different priority level.

\section{REFERENCES}

[1] B. Braden, D. Clark, J. Crowcroft, et al. Recommendations on queue management and congestion avoidance in the Internet. RFC2309, Apr.1998

[2] T.V. Lakshman, A. Neidhardt, T. Ott. The drop from front strategy in TCP over ATM and its interworking with other control features. In Proc. INFOCOM 96, 1996

[3] S. Floyd, V. Jacobson. Random early detection gateways for congestion avoidance. IEEE/ACM Trans. Networking, Aug.1993,1(4):397-413.

[4] S. Floyd, RED with drop from front, Mar. 1998, ftp://ftp.ee.lbl.gov/email/sf.98mar11.txt

[5] J. Padhye, et al. Modeling TCP throughput: a simple model and its empirical validation. In Proc. SIGCOMM 98, 1998.

[6] S. Floyd, K. Fall. Promoting the use of end-to-end congestion control in the Internet. IEEE/ACM Trans. Networking, Aug.1999,7(4): 458-472.

[7] B. Suter, T. V. Lakshman, D. Stiliadis, et al. Buffer management schemes for supporting TCP in gigabit routers with per-flow queueing. IEEE J-SAC, Jun.1999,17(6):1159-1169.

[8] Stoica, S. Shenker, H. Zhang. Core-stateless fair queueing: Achieving approximately fair bandwidth allocations in high speed networks. In Proc. ACM SIGCOMM'98, 1998

[9] M.J. Karam, F. Tobagi. Rate and queue controlled random drop (RQRD): A buffer management scheme for Internet routers. In Proc. Globecom'00, 2000

[10] NS (network simulator). Available: http://www.isi.edu/nsnam/ns/

[11] V. Paxson, S. Floyd. Wide area traffic: the failure of Poisson modeling. IEEE/ACM Trans. Networking, Jun.1995,3(3):226-244.

[12] M. Crovella, A. Bestavos. Self-similarity in World Wide Web traffic: evidence and possible causes. In Proc. SIGMETRICS 96, 1996.

[13] W. Willinger, M. Taqqu, R. Sherman, et al. Self-similarity through high-variability: statistical analysis of Ethernet LAN traffic at the source level. IEEE/ACM Trans. Networking, Feb.1997, 5(1):71-86. 


\section{TrAFFiC ENGINEERING AND DIFFSERV}

